\title{
Exposure to a chronic high-fat diet promotes atrial structure and gap junction remodeling in rats
}

\author{
TIANYU MENG ${ }^{1}$, GONG CHENG $^{2}$, YUEJIAO WEI ${ }^{1}$, SHUTING MA $^{1}$, YONGRONG JIANG ${ }^{1}$, \\ JINE WU ${ }^{1}, \mathrm{XIN}_{\mathrm{ZHOU}}{ }^{1}$ and CHAOFENG SUN ${ }^{1,3}$ \\ ${ }^{1}$ Department of Cardiovascular Medicine, The First Affiliated Hospital of Xi'an Jiaotong University, Xi'an, Shaanxi 710061; \\ ${ }^{2}$ Department of Cardiovascular Medicine, Shaanxi Provincial People's Hospital, Xi'an, Shaanxi 710068; \\ ${ }^{3}$ Shaanxi Key Laboratory of Molecular Cardiology, Xi'an Jiaotong University, Xi'an, Shaanxi 710061, P.R. China
}

Received August 28, 2016; Accepted May 5, 2017

DOI: $10.3892 /$ ijmm.2017.2982

\begin{abstract}
Obesity has been demonstrated to be linked to atrial fibrillation (AF) with atrial enlargement and tissue fibrosis. Long-term high calorie intake is the main reason for the prevalence of obesity. To investigate the possible causes of AF, such as chronic high-fat diet (HFD), and to identify the underlying mechanisms, the present study analyzed a variety of structural and gap junctional electrophysiological alterations in the atria of female rats fed an HFD. After consistent HFD feeding of female rats for 12 weeks, hematoxylin and eosin (H\&E) and Masson's staining, RT-qPCR, western blotting, immunofluorescence and TUNEL staining were performed. In our study, approximately $3 / 5$ of the HFD-fed rats (HFD-OB, $n=13$ ) displayed a significant increase in body weight, while the other $2 / 5$ did not (HFD-NOB, $n=8$ ). In addition, the atrial weight of the HFD-OB and HFD-NOB rats was markedly heavier, as compared to the rats fed a normal diet $(C T, n=20)$. According to the plasma lipid levels, both HFD-OB and HFD-NOB rats exhibited dyslipidemia. Furthermore, H\&E staining revealed broadened interstitial space and myocyte disarray in atria of the HFD-fed rats (i.e., HFD-OB and HFD-NOB rats). Expression levels of atrial fibrosis relevant factors, transforming growth factor- $\beta 1$ and matrix metalloproteinase- 2 , were significantly upregulated in the HFD-fed rat atria. In addition, we found a gap junction remodeling with distinct alterations in expression and distribution of connexin $40(\mathrm{Cx} 40)$ and $\mathrm{Cx} 43$ in the HFD-fed rat atria. Moreover, a modest increase in apoptotic cell death in both the HFD-OB and HFD-NOB rat atria was detected. Taken together, our findings demonstrated that the
\end{abstract}

Correspondence to: Professor Chaofeng Sun, Department of Cardiovascular Medicine, The First Affiliated Hospital of Xi'an Jiaotong University, 277 West Yanta Road, Xi'an, Shaanxi 710061, P.R. China

E-mail: csun1@163.com

Key words: high-fat diet, susceptibility to atrial fibrillation, obesity, transforming growth factor- $\beta 1$, matrix metalloproteinase-2, connexin 40 , connexin 43 , apoptosis impact of chronic HFD on atria displayed in the diet-induced obese rats was observed in HFD-fed rats in the absence of obesity as well.

\section{Introduction}

Atrial fibrillation (AF) is the most common supraventricular arrhythmia in clinical practice and is considered to be a growing cardiovascular epidemic (1). Emerging evidence demonstrates that aging alone does not account for the standing increase in AF prevalence (2). Obesity has been characterized as a new risk factor contributing to AF (3). Obesity is a global pandemic with more than $2 / 3$ adults being overweight or obese $(4,5)$. It is well-documented that a long-term high calorie intake and sedentary lifestyle are the underlying causes of the high prevalence of obesity (6,7). Exposure to a chronic high-fat diet (HFD) is not only linked to diabetes, it also has a strong association with cardiovascular disease and stroke (7-9). Although analysis of numerous studies over the last decades has displayed the effect of obesity on atrial structural (10-14) and gap junctional [mainly focused on connexin $43(\mathrm{Cx} 43)$ expressed and distributed in ventricles] remodeling $(15,16)$, the mechanisms remain incompletely elucidated. Despite the unequivocal linkage between obesity and AF, it remains currently unknown whether structural and gap junctional remodeling following a chronic HFD feeding and/or increased AF risks are prevalent in the absence of HFD-induced obesity. Therefore, our study analyzed the structural and gap junctional electrophysiological alterations in the atria of female rats fed a consistent HFD for 12 weeks, determined the impact of HFD on the susceptibility to AF, investigated whether this effect may be the same in both HFD-induced obesity (HFD-OB) and HFD-fed non-obesity (HFD-NOB) rats, and aimed at identifying the underlying mechanisms.

\section{Materials and methods}

Experimental animals. The female Sprague-Dawley (SD) rats used in this study were obtained from the Laboratory Animal Center of Xi'an Jiaotong University (Xi'an, China). Rats were housed in an animal research facility with a 12-h light/dark cycle at $20-25^{\circ} \mathrm{C}$ room temperature and given 
free access to food and water. Ethical approval of this study was obtained from the Ethics Committee of Xi'an Jiaotong University. Animal experiments involved in this study were performed according to the guidelines of Animal Handling and Experimentation (Xi'an, China).

HFD feeding and grouping. HFD-fed rats $(\mathrm{n}=21)$ were provided with an HFD consisting of $60 \%$ of calories from fat (Research Diets, Inc., New Brunswick, NJ, USA; Laboratory Animal Center of Xi'an Jiaotong University) starting at 6-8 weeks of age for 12 weeks. Control rats $(\mathrm{CT}, \mathrm{n}=20)$ were fed a normal diet (ND) consisting of $4.5 \%$ fat. Weight measure was performed and recorded per week. After 12 weeks of HFD feeding, HFD-fed rats were divided into HFD-OB and HFD-NOB according to the levels of weight increase.

Plasma lipid measurements. Rats were anaesthetized with $10 \%$ chloral hydrate. Right after abdomen opening, 5 to $10 \mathrm{ml}$ of blood was drawn from the abdominal aortae and collected with heparinized anticoagulation tubes and immediately centrifuged at $15,000 \mathrm{rpm}$ for $30 \mathrm{~min}$ at $4^{\circ} \mathrm{C}$, and then the plasma was stored at $-80^{\circ} \mathrm{C}$. Samples were sent to the Clinical Laboratory Department of the First Affiliated Hospital of Xi'an Jiaotong University to test the levels of total cholesterol (TC), high-density lipoprotein cholesterol (HDL-C), low-density lipoprotein cholesterol (LDL-C) and triglycerides (TGs).

Tissue harvesting and processing. After piercing the left ventricle and carving the right atrium, the heart was perfused with phosphate-buffered saline (PBS) injected into the left ventricle, vessels were rinsed subsequently and both atria were harvested after thoroughly removing aorta and fat. For histological analysis, atrial tissue was fixed in $4 \%$ paraformaldehyde dissolved in PBS, dehydrated and embedded in paraffin. For immunofluorescence assay, atrial tissue was washed with PBS, fixed in $4 \%$ paraformaldehyde, dehydrated with sucrose, embedded in optimal cutting temperature (OCT) compound, and then kept at $-80^{\circ} \mathrm{C}$ for long-term storage. For biochemical examinations, atrial tissue was transiently frozen in liquid nitrogen and stored at $-80^{\circ} \mathrm{C}$.

Hematoxylin and eosin (H\&E) and Masson's staining. Processing of rat atrial tissue paraformaldehyde-fixing and paraffin-embedding has been previously described. Sections $(5-\mu \mathrm{m}$ thick) from each atrial tissue were stained with $\mathrm{H} \& \mathrm{E}$ or Masson's staining, and then sealed and stored at $4^{\circ} \mathrm{C}$. Atrial fibrosis was assessed using Image-Pro Plus 6.0 analysis software (Media Cybernetics, Inc., Rockville, MD, USA).

RNA extraction and reverse transcription-quantitative $P C R(R T-q P C R)$. Total RNA was extracted from rat atrial tissue using the TRIzol reagent (Invitrogen Life Technologies, Carlsbad, CA, USA) in accordance with the manufacturer's instructions, and then reverse transcribed using the RevertAid First Strand cDNA synthesis kit (Thermo Fisher Scientific, Inc., Waltham, MA, USA) for $60 \mathrm{~min}$ at $42^{\circ} \mathrm{C}$ and $5 \mathrm{~min}$ at $80^{\circ} \mathrm{C}$. Quantitative PCR (qPCR) was performed using the FastStart Universal SYBR-Green Master (Rox) (Roche Diagnostics, Indianapolis, IN, USA) on the $\mathrm{iQ}^{\mathrm{TM}}$ Multicolor Real-Time PCR detection system (Bio-Rad Laboratories, Inc., Hercules,
CA, USA). The thermocycling parameters of PCR were $10 \mathrm{~min}$ at $95^{\circ} \mathrm{C}, 40$ cycles at $95^{\circ} \mathrm{C}$ for $15 \mathrm{sec}$ and $60^{\circ} \mathrm{C}$ for $60 \mathrm{sec}$, followed by a melting curve stage. Each atrial sample was performed in triplicate. The primer sequences used for rat atrial $\mathrm{Cx} 40$ were forward, 5'-CAG GCA GAT TTC CAG TGT GAT-3' and reverse, 5'-AGT AGC GGA TGT GAG AGA TGG-3'; rat atrial Cx43 forward, 5'-GCT GTG TCC TTG GTG TCT CTT G-3' and reverse, 5'-ACA GCT TTT GGA GGG GCT CAG T-3'; rat atrial transforming growth factor- $\beta 1$ (TGF- $\beta 1$ ) forward, 5'-AAC CCA CAA CGA AAT CTA TGA CAA-3' and reverse, 5'-AGA GCA ACA CGG GTT CAG GTA-3'; and rat atrial matrix metalloproteinase-2 (MMP-2) forward, 5'-CAG GCT CTT CTC CTT TCA CAA C-3' and reverse, 5'-AAG CCA CGG CTT GGT TTT CCT C-3'. Target mRNAs mentioned above were normalized to the GAPDH mRNA level.

Western blotting. The rat atrial tissue was sufficiently ground and lysed within a tissue homogenizer supplemented with RIPA buffer and protease inhibitors (100:1), laid on ice for $15 \mathrm{~min}$, and then centrifuged at $15,000 \mathrm{rpm}$ for $30 \mathrm{~min}$ at $4^{\circ} \mathrm{C}$. Supernatant liquid removed from each sample was kept. The total protein concentration was determined using BCA protein assay reagent (Pierce, Rockford, IL, USA). After being standardized by SDS-PAGE sample loading buffer and boiled for $5 \mathrm{~min}$, equivalent amounts $(30 \mu \mathrm{g})$ of protein extracted from each sample were separated onto a $10 \%$ SDS-PAGE gel for electrophoresis. Then proteins were transferred onto polyvinylidene difluoride (PVDF) membranes (Millipore, Bedford, MA, USA) under the condition of $200 \mathrm{~mA}$ for $1 \mathrm{~h}$. The blotted membranes were incubated in blocking buffer (5\% defatted milk in TBS containing $0.1 \%$ Tween-20) for $1 \mathrm{~h}$ at room temperature (RT). Then the blots were probed overnight at $4{ }^{\circ} \mathrm{C}$ with the following primary antibodies: anti-TGF- $\beta 1$ (ab92486; Abcam, Cambridge, UK), anti-Cx43 (3512S), anti-MMP-2 (4022S) (Cell Signaling Technology Inc., Danvers, MA, USA), anti-Cx40 (sc-20466), and anti-GAPDH (sc-25778) (Santa Cruz Biotechnology, Inc., Santa Cruz, CA, USA). After completion of all the procedures mentioned above, incubation with goat-anti-rabbit horseradish peroxidase (HRP)-conjugated IgG as the secondary antibody was carried out for $1 \mathrm{~h}$ at RT. The blot bands were visualized using ECL and quantified using Quantity One analysis software (both from Bio-Rad Laboratories, Inc.).

Immunofluorescence and TUNEL staining. Cryosections $(6-\mu \mathrm{m}$ thick) of atrial tissue were performed after paraformaldehydefixing, dehydrating and OCT-embedding, washed with PBS for $5 \mathrm{~min}, 3$ times, followed by incubation overnight at $4^{\circ} \mathrm{C}$ with the primary antibodies as follows: goat polyclonal anti-Cx40 (1:200 to 1:500; ab16585; Abcam), rabbit polyclonal anti-Cx43 (1:400; 3512S; Cell Signaling Technology, Inc.). Secondary antibodies involved in the present study were fluorescein isothiocyanate (FITC)-conjugated AffiniPure goat anti-rabbit and donkey anti-goat IgG (H+L) (1:200; ZSGB-Bio Co., Ltd., Beijing, China). Nuclei were stained at RT for 15 min using 4'-6-diamidino-2-phenylindole (DAPI; Bioworld Technology, Inc., St. Louis Park, MN, USA). Cell death was assessed using a terminal deoxynucleotidyl transferase-mediated dUTP nick end labelling (TUNEL) kit (Roche Diagnostics, Rotkreuz, Switzerland). 
Statistical analysis. Statistical data are expressed as mean \pm SD. To compare the results between different groups, an unpaired Student's t-test or analysis of variance (ANOVA) with post hoc Dunnett's test was used. A P-value $<0.05$ was considered statistically significant.

\section{Results}

Female $S D$ rats fed an HFD exhibit differences in weight increase. To determine the role of HFD in AF, we analyzed the 6- to 8-week-old SD rats fed an HFD or ND. The body weight showed no significant difference among these groups until the 4th week. After 12 weeks of feeding, only $~ 3 / 5$ of the HFD-fed rats (HFD-OB, $n=13$ ) displayed a significantly higher body weight increase than the rest of the HFD-fed rats (HFD-NOB, $n=8$ ) and those fed with an ND (CT, $\mathrm{n}=20$ ) (equal as week 0; Fig. 1A). Furthermore, compared with the HFD-NOB rats, HFD-OB rats were heavier which was not due to the differences in food intake (data not shown). In comparison to the $\mathrm{CT}$ rats, no significant difference in weight increase was shown in the NOB rats. Moreover, the analysis revealed markedly increased atrial weight in the HFD-fed rats (Fig. 1B). As shown in Fig. 1B, there was a marked increase in atrial weight in the HFD-NOB rats, as compared to that noted in the HFD-OB rats.

Both HFD-OB and HFD-NOB rats exhibit dyslipidemia. Metabolic disorders play a principal role in the incidence, maintenance and recurrence of AF. To examine the status of lipid metabolism in HFD-fed rats, we measured the plasma lipid levels. HFD-NOB $(n=8)$ rats displayed a marginal elevation in TG level, as well as significantly upregulated levels of TC and LDL-C, as compared to the CT rats $(n=20)$ (Fig. 2). Although TG, TC and LDL-C levels were all markedly increased in the HFD-OB rats ( $\mathrm{n}=13)$, TG, a key lipid metabolic factor, was not significantly elevated, suggesting that not all factors participated in the alteration of the lipid metabolism in the HFD-NOB rats. The HDL-C levels did not reveal any difference among the three groups. The results indicated a disturbed lipid homeostasis in the HFD-fed rats.

HFD-fed rats exhibit more extensive atrial fibrosis. Atrial fibrosis is well described as an AF-promoting condition and a potential predictor to recurrence (17). Tissue fibrosis is mediated by a series of risk factors, such as age, sex, heart failure, stroke and hypertension. We performed histological assay (H\&E and Masson's staining) to confirm the association between HFD and atrial fibrosis. Indeed, the H\&E staining of atrial paraffin sections revealed more broadened interstitial space among the atrial myofibers as well as distinctly disordered layout of atrial myocytes in both the HFD-OB and HFD-NOB rats instead of a normal myocardial structure (Fig. 3). H\&E staining of atrial tissue of the OB and NOB rats did not exhibit any difference. Significant fibrosis was shown in Masson's staining of the HFD-OB and HFD-NOB rat atrial sections (Fig. 4). Extensive fibrosis was particularly visible around atrial myocytes and was disorderly distributed. In contrast, the tiny amounts of collagen fibers in the $\mathrm{CT}$ rat atria were continuous and complete. As shown in Fig. 4B, compared with the HFD-OB rats, the collagen area of the HFD-NOB rat atria was larger, while there was no
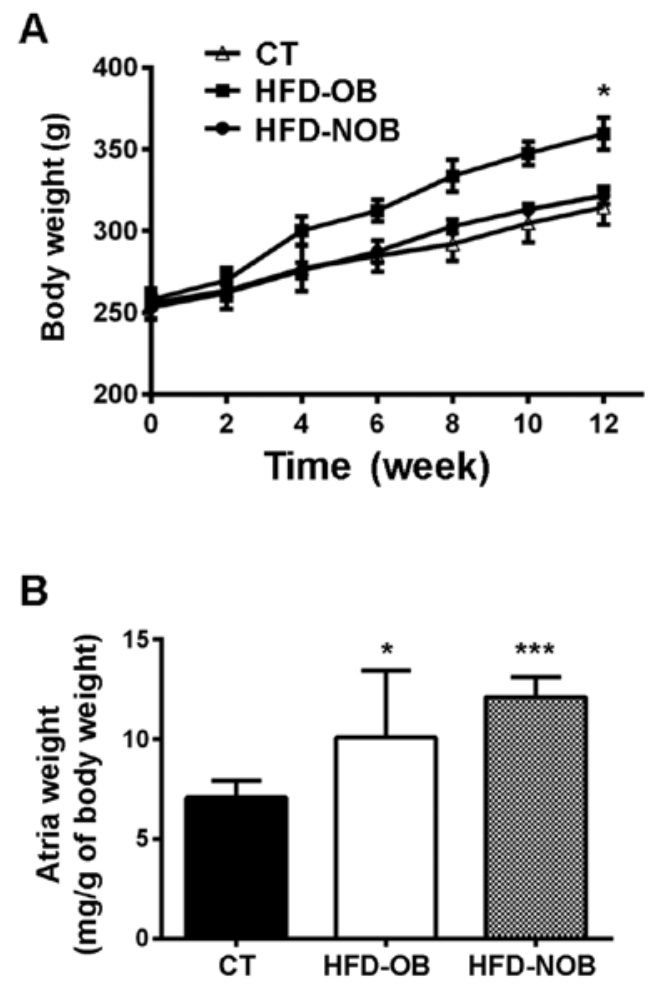

Figure 1. Measurements of body weight and atrial weight in female SD rats fed a normal diet (CT) or high-fat diet (HFD). (A) Body weight increase. HFD feeding started at 6-8 weeks of age (time-point, 0). (B) Atrial weight was measured and normalized to body weight. Data are expressed as mean $\pm \mathrm{SD}$. ${ }^{*} \mathrm{P}<0.05$ vs. control (CT). ${ }^{* * *} \mathrm{P}<0.001$ vs. $\mathrm{CT}, 12$ weeks after HFD feeding. HFD-OB, HFD-fed obese rats; HFD-NOB, HFD-fed non-obese rats.

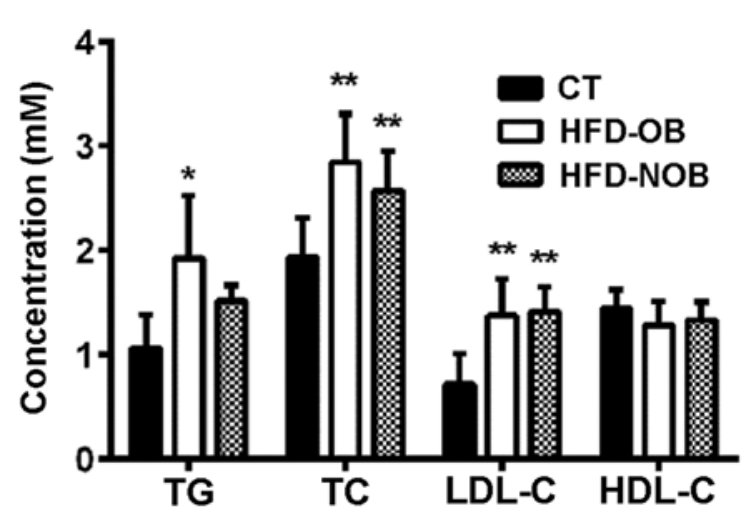

Figure 2. High-fat diet-induced obesity (HFD-OB) and HFD-fed nonobesity (HFD-NOB) rats exhibit dyslipidemia. Plasma triglyceride (TG), total cholesterol (TC), low-density lipoprotein cholesterol (LDL-C) and high-density lipoprotein cholesterol (HDL-C) levels were determined from independent samples of control (CT), HFD-OB and HFD-NOB rats (means $\pm \mathrm{SD}$. ${ }^{*} \mathrm{P}<0.05$ vs. $\mathrm{CT} ;{ }^{* *} \mathrm{P}<0.01$ vs. $\mathrm{CT}$ ). HFD-OB, HFD-fed obese rats; HFD-NOB, HFD-fed non-obese rats

significant difference between these two groups. These results above revealed that HFD may somehow induce and promote atrial fibrosis. The impacts of HFD on atrial fibrosis exhibited in the HFD-OB and HFD-NOB rats were similar.

HFD feeding upregulates expression of mRNA and proteins related to atrial fibrosis. Previous studies demonstrated 

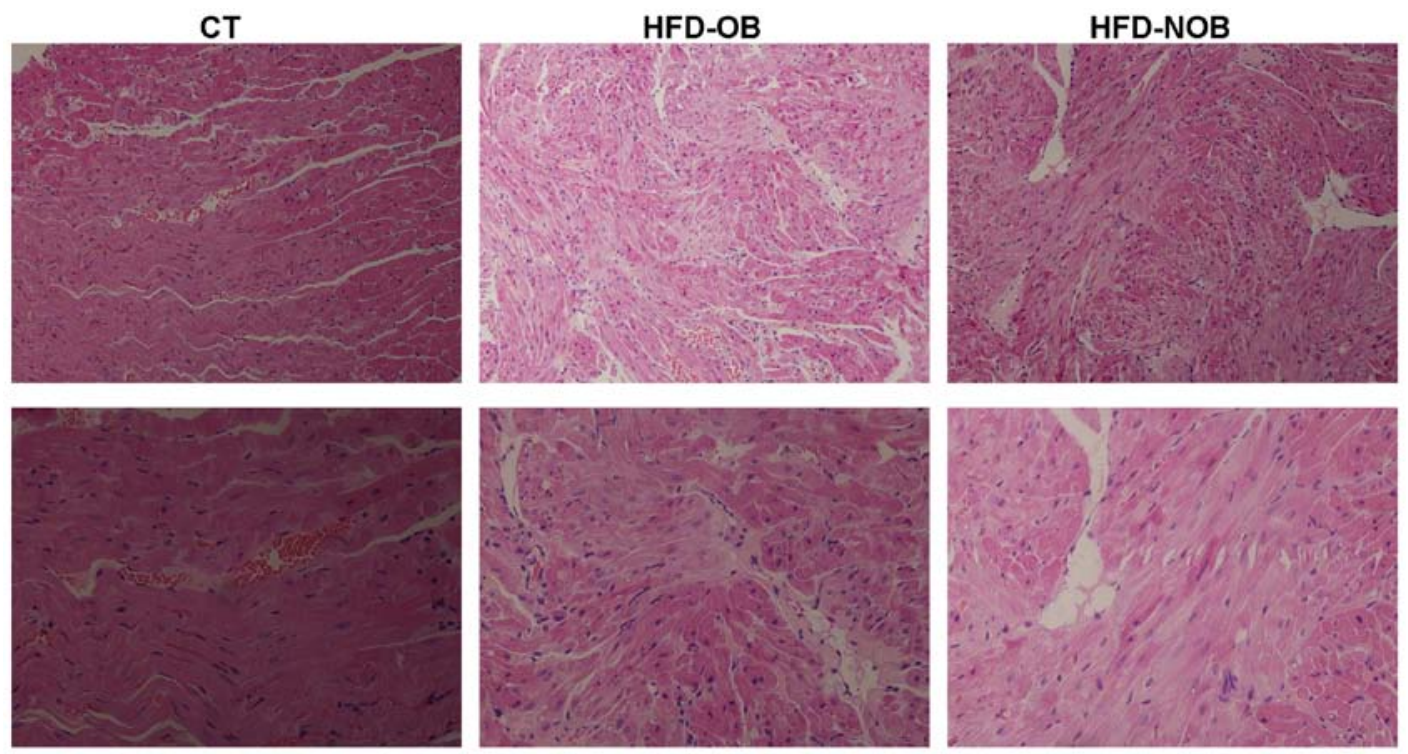

Figure 3. Hematoxylin and eosin (H\&E) staining of atrial sections from rats fed a normal diet (ND) or a high-fat diet (HFD). Atrial myofibers were stained in red, while nuclei were stained purple. Control (CT) rats displayed a complete and regular atrial myocardium structure. In contrast, atrial myofibers were disorderly arrayed in the HFD-fed obese (HFD-OB) and HFD-fed non-obese (HFD-NOB) rats. Representative images are at a magnification, x100 (top panels) and $\times 200$ (bottom panels).

A
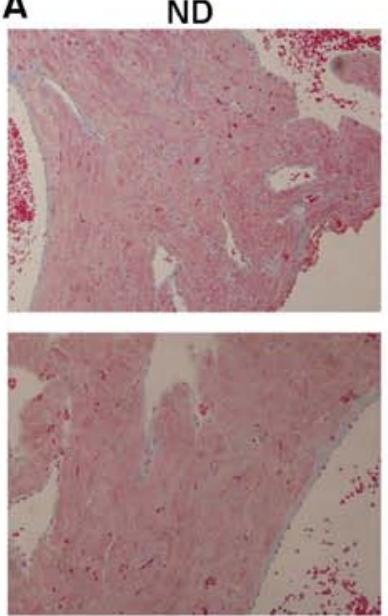
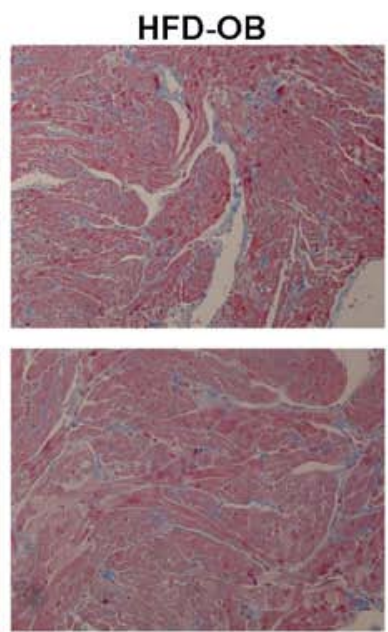
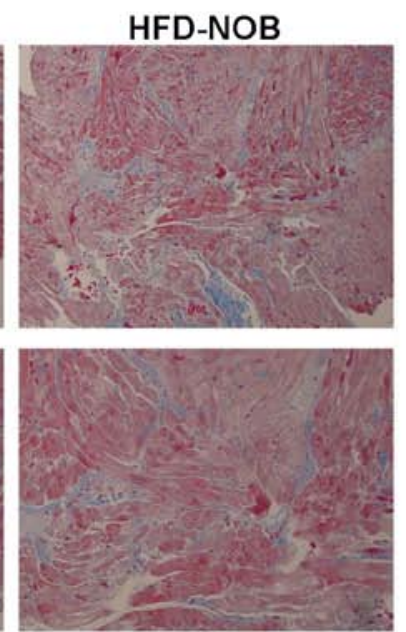

B

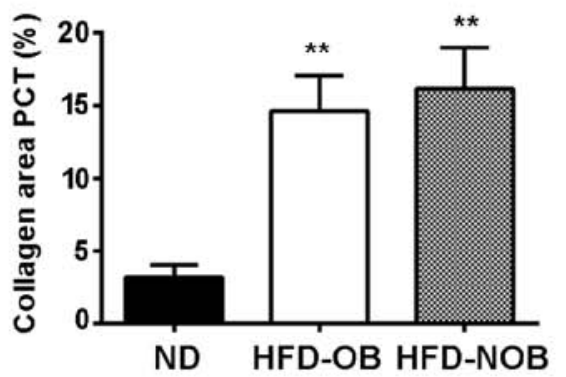

Figure 4. Masson staining and semi-quantitative assessment of collagen. (A) Atrial myocytes were stained in red, while collagen fibers were stained in blue-purple. High-fat diet (HFD)-fed rat atria exhibited large amounts of collagen fibers in chaos, as compared to normal diet (ND)-fed rat atrial tissue. Representative images are at a magnification, x100 (top panels) and x200 (bottom panels). (B) Atrial tissue of HFD-fed rats displayed larger collagen area. Results are expressed as means $\pm \mathrm{SD},{ }^{* *} \mathrm{P}<0.01$ vs. control (CT). HFD-OB, HFD-fed obese rats; HFD-NOB, HFD-fed non-obese rats.

that TGF- $\beta 1$, a multifunctional cytokine participating in the regulation of cell proliferation and differentiation, leads to atrial fibrosis and AF (18). In addition, MMP-2, playing a key role in extracellular matrix activity, promotes atrial fibrosis and is upregulated during AF (19). We performed further biochemical analyses to explore the association between HFD and atrial fibrosis. RT-qPCR analysis of TGF- $\beta 1$ and MMP-2 mRNA revealed that the expression of TGF- $\beta 1$ and MMP-2 mRNA was significantly upregulated in the HFD-OB and HFD-NOB rat atrial myocytes (Fig. 5A and B). No significant difference was observed between these two groups. In accordance with the results of the RT-qPCR analysis, western blotting bands showed a significant increase in expression of TGF- $\beta 1$ and MMP- 2 protein in the atria of rats fed an HFD as well (Fig. 5C and D). Similarly, the increases showed no significant difference between the HFD-OB and HFD-NOB groups. The marked increases in expression of TGF- $\beta 1$ and MMP-2 proteins and mRNA further implied that HFD may induce tissue fibrosis in the atria. In addition, the levels of atrial fibrosis-promoting factors expressed in the HFD-OB and HFD-NOB rats showed no significant difference.

HFD feeding downregulates expression of gap junction $m R N A$ and proteins. Abnormality in expression/distribution of gap junction Cxs is one of the essential components of atrial electrical remodeling. $\mathrm{Cx} 40$ and $\mathrm{Cx} 43$ are known to be the most commonly expressed $\mathrm{Cx}$ types in atrial myocytes. Thus, for the purpose of elucidating the potential mechanisms 
A

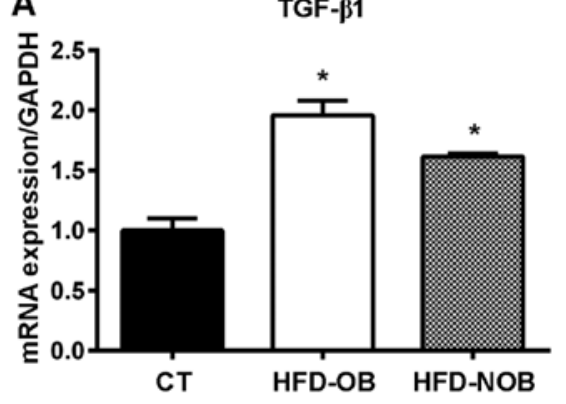

C
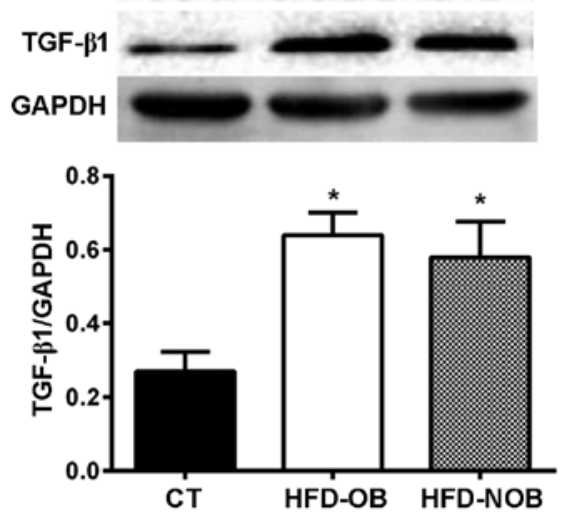

B $\quad$ MMP-2

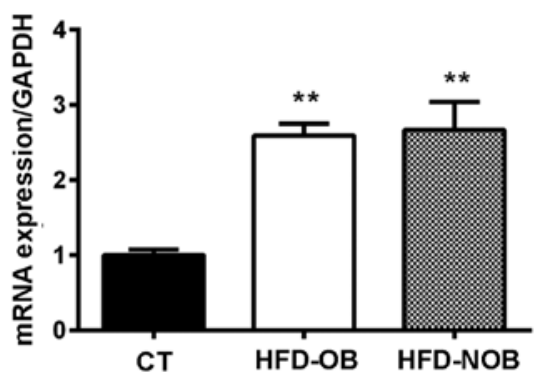

D
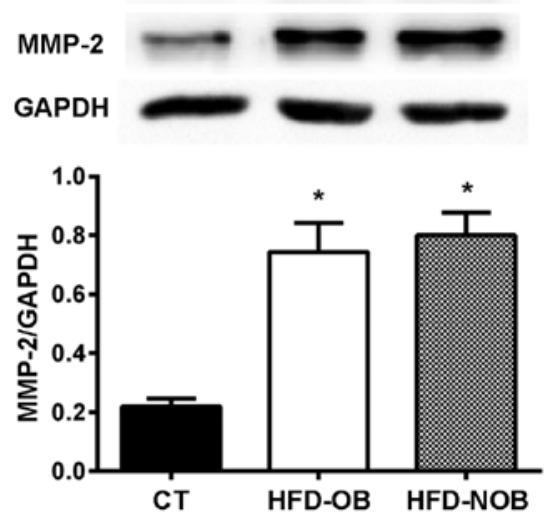

Figure 5. mRNA and protein expression of transforming growth factor- $\beta 1$ (TGF- $\beta 1$ ) and matrix metalloproteinase-2 (MMP-2) in atria obtained from rats fed a normal diet (ND) or a high-fat diet (HFD). (A and B) RT-PCR analysis of TGF- $\beta 1$ and MMP-2. Relative mRNA levels were normalized to GAPDH. $(\mathrm{C}$ and $\mathrm{D})$ Representive western blots and corresponding quantification showing TGF- $\beta 1$ and MMP-2 proteins in atria. Results are expressed as means \pm SD, "P<0.05 vs. control (CT); *"P $<0.01$ vs. CT. HFD-OB, HFD-fed obese rats; HFD-NOB, HFD-fed non-obese rats.
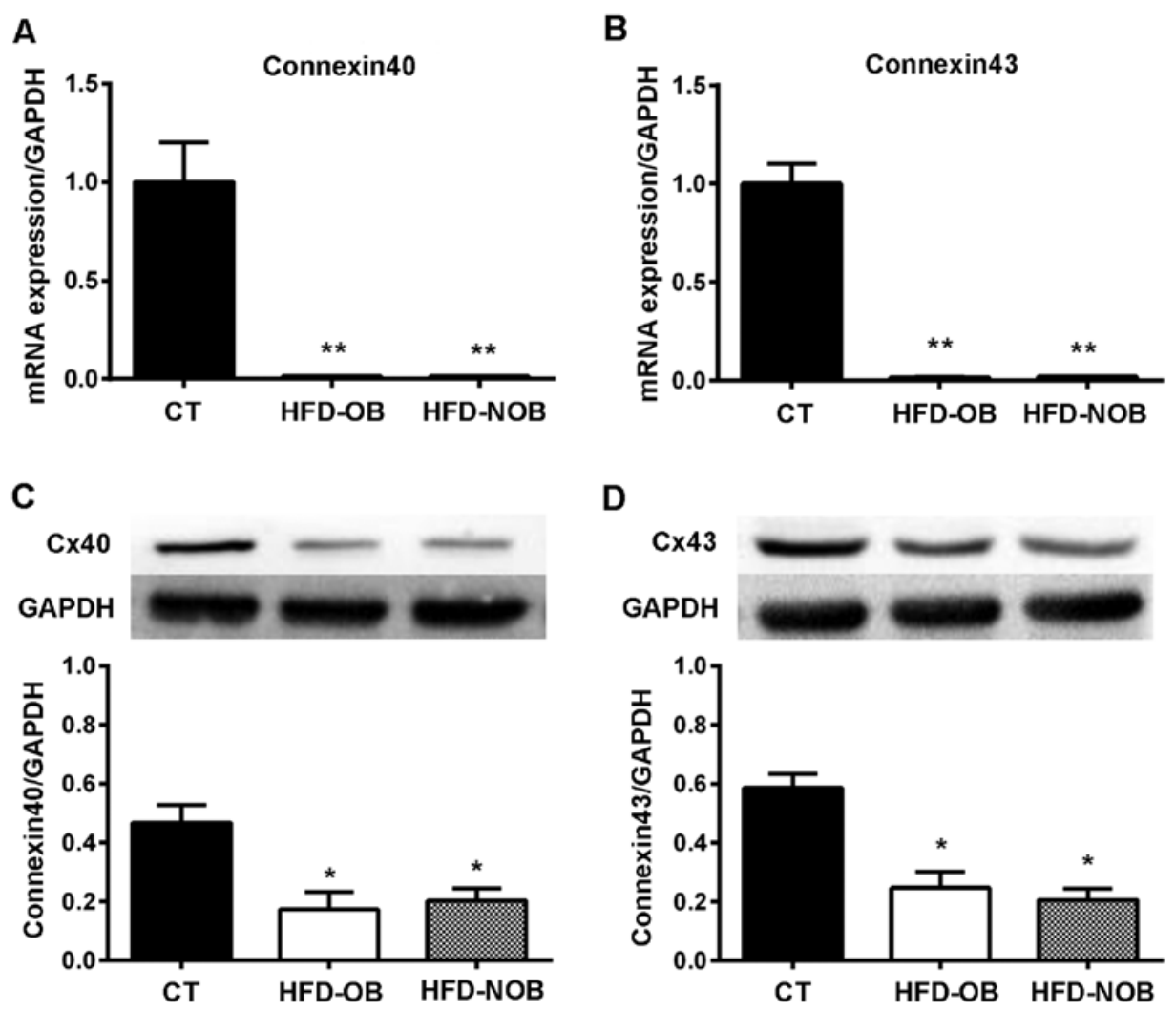

Figure 6. RT-PCR and western blotting of connexin40 (Cx40) and $\mathrm{Cx} 43$ in atria obtained from rats fed a normal diet (ND) or a high-fat diet (HFD). (A and B) Relative mRNA expression of $\mathrm{Cx} 40$ and Cx43. mRNA levels were normalized to GAPDH. (C and D) Representive western blots and corresponding quantification showing $\mathrm{Cx} 40$ and $\mathrm{Cx} 43$ proteins in atria. Values are presented as means $\pm \mathrm{SD},{ }^{*} \mathrm{P}<0.05$ vs. control (CT); ${ }^{* *} \mathrm{P}<0.01 \mathrm{vs}$. CT. HFD-OB, HFD-fed obese rats; HFD-NOB, HFD-fed non-obese rats. 

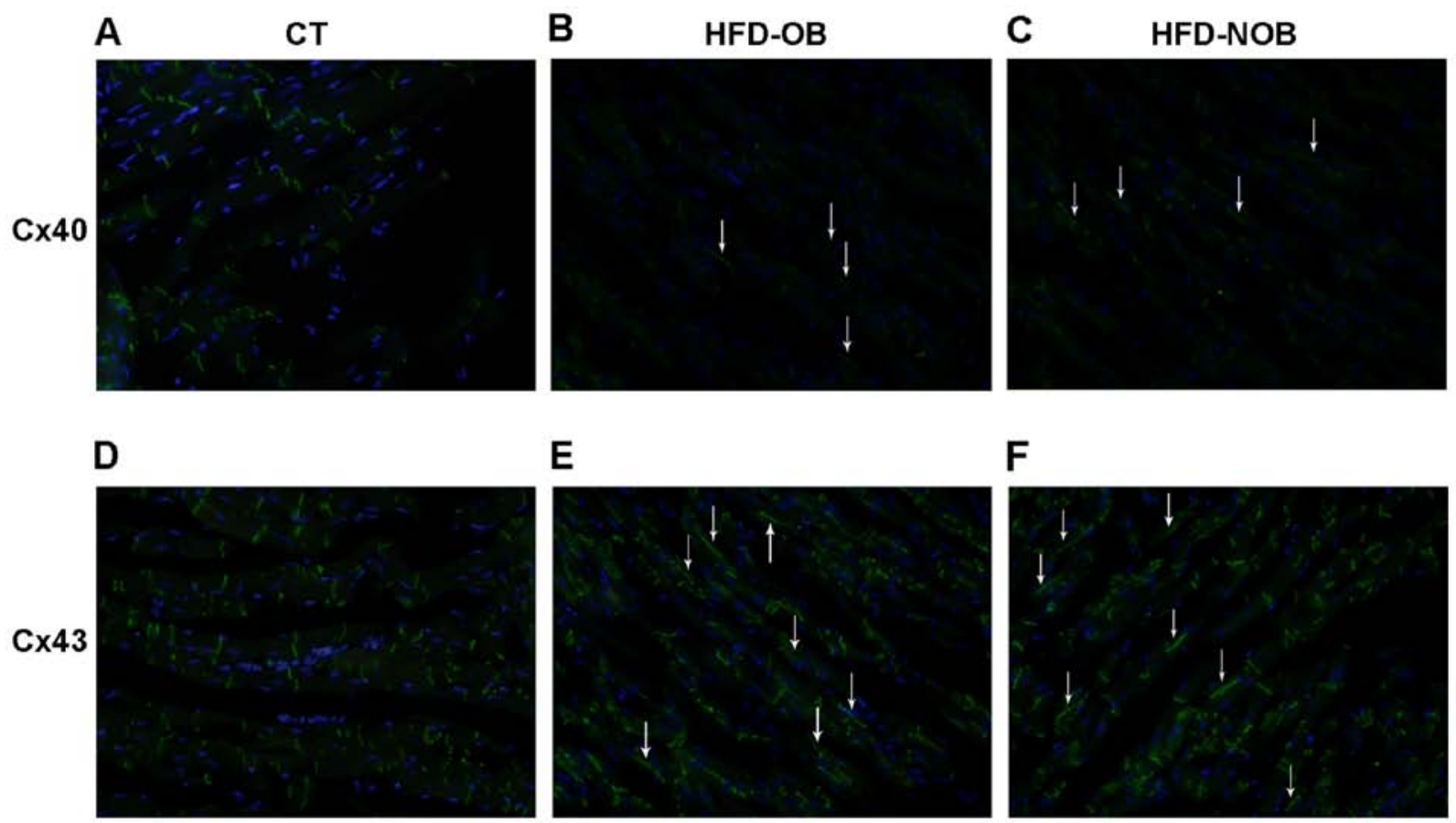

E

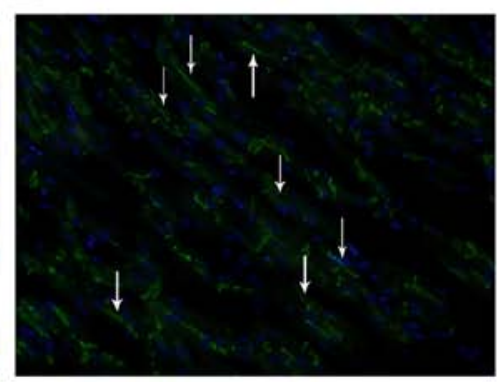

$\mathbf{F}$

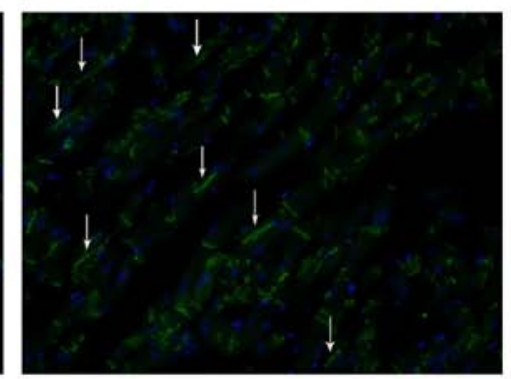

Figure 7. Immunofluorescence staining exhibits atrial gap junction remodeling in rats fed a normal diet (ND) or a high-fat diet (HFD). (A and D) Modest connexin40 (Cx40) and Cx43 immune signals were detected at the intercalated discs of atrial myocytes in the control (CT) rat atria. (B and C) $\mathrm{Cx}$ staining was significantly decreased in the atria of rats fed an HFD, especially the staining of Cx40. (E and F) In the HFD-fed obese (HFD-OB) and HFD-fed non-obese (HFD-NOB) rat atria, $\mathrm{Cx} 43$ distribution was observed to be markedly lateralized (arrows indicating lateralized distribution of $\mathrm{Cx} 40$ and $\mathrm{Cx} 43$ ).

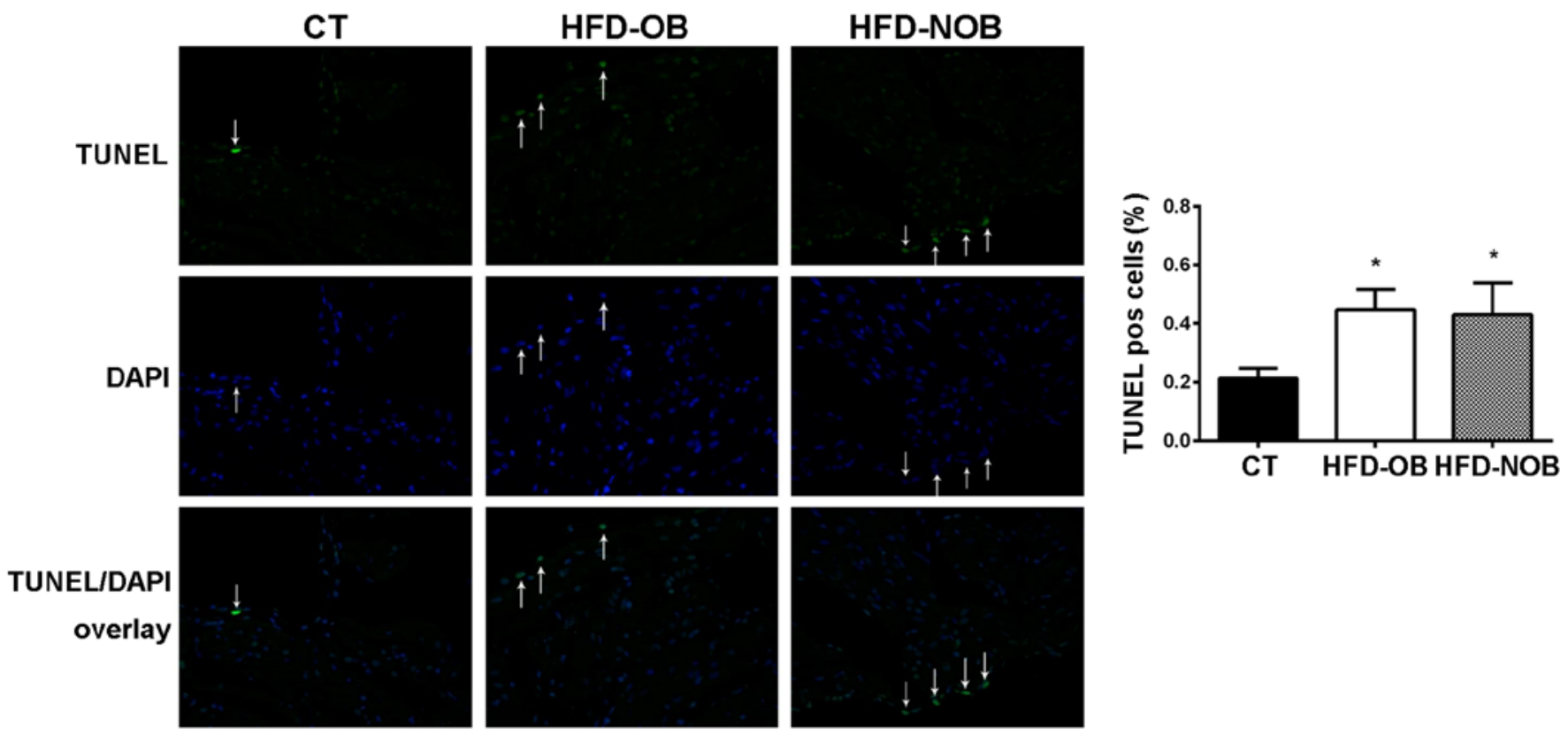

Figure 8. TUNEL staining of atrial sections from rats fed a normal diet (ND) or a high-fat diet (HFD). Arrows denote TUNEL-positive nuclei and corresponding DAPI staining and quantification is shown (right graph). Data are expressed as mean $\pm \mathrm{SD},{ }^{*} \mathrm{P}<0.05$ vs. control (CT). HFD-OB, HFD-fed obese rats; HFD-NOB, HFD-fed non-obese rats.

accounting for the atrial remodeling-promoting effects of HFD, we measured the expression levels of Cx40 and Cx43 and their mRNA levels using western blotting and RT-qPCR analysis respectively. RT-qPCR analysis displayed a significant downregulation in the expression of $\mathrm{Cx} 40$ and $\mathrm{Cx} 43$ mRNA in the HFD-fed rat atrial tissue (Fig. 6A and B). In addition, a similar marked decrease in the protein expression of Cx40 and Cx43 was observed by western blotting as well (Fig. 6C and D). Neither the RT-qPCR nor the western blotting showed significant differences between the HFD-OB and HFD-NOB groups. The altered expression levels of gap junction CXs suggest that an HFD could impact the electrical 
remodeling to a certain extent, which may further provide the substrate susceptible to AF.

HFD-fed rats show altered distribution of gap junction in the atria. Previous studies have demonstrated an association between the abnormal distribution of gap junction and arrhythmogenesis $(22,28)$. Further analysis using immunofluorescence exhibited altered expression and distribution of $\mathrm{Cx} 40$ and $\mathrm{Cx} 43$ in atrial myocytes of the rats fed an HFD, as compared to the ND-fed rats (Fig. 7). As shown in Fig. 7A and D, Cx40 and Cx43 were mainly located at the intercalated discs of atrial myocytes in the $\mathrm{CT}$ rats. $\mathrm{Cx} 40$ protein expression displayed significant downregulation in the HFD rat atrial myocytes via western blotting, supporting the results of the immunofluorescence approach (Fig. 7B and C). In addition, $\mathrm{Cx} 40$ was showed to be laterally distributed along the longitudinal atrial myocyte membranes. Cx43 was markedly downregulated and laterally distributed in the HFD-OB rat atria as well (Fig. 7E). Nevertheless, in comparison to the $\mathrm{CT}$ rats, $\mathrm{Cx} 43$ signals seemingly did not show a significant decrease in the HFD-NOB rats (Fig. 7F). Although lateralized distribution of $\mathrm{Cx} 43$ was still observed in the HFD-NOB rat atrial myocytes, these alterations in expression and distribution of gap junction revealed that it may be more susceptible to $A F$ via $H F D$-induced $C x$ remodeling. In addition, $H F D-O B$ and HFD-NOB rats showed similar levels of gap junctional remodeling induced by HFD.

HFD rat atrial tissue displays a tendency of increased apoptotic cell death. To highlight the effects of HFD on the atrial substrate, we therefore quantified cell death in serial sections of atrial tissue in the ND and HFD rats. Cell death was markedly lower in the rats fed an ND, whereas there was no significant difference observed between the HFD-OB and HFD-NOB rats (Fig. 8).

Taken together, these data demonstrated that whether or not HFD induces obesity, HFD may affect lipid metabolism, alter the expression of mRNA and proteins involved in atrial fibrosis and promote the remodeling of gap junction in rat atrial tissue. The impact of HFD on the atrial substrate may represent the underlying cause for the incidence, maintenance and recurrence of AF.

\section{Discussion}

In the present study, 6- to 8-week-old female SD rats were fed an HFD for 12 weeks, and subsequent atrial fibrosis and gap junction remodeling were analyzed. Large numbers of previous studies have reported significant increases in the body weight of HFD-fed rats $(20,21)$. However, our findings showed that only approximately $3 / 5$ of the HFD-fed rats were more susceptible to HFD-induced obesity, while the other $2 / 5$ were not (Fig. 1A). We considered that the distinction in body weight increases may be due to the genetic variations in female SD rats. Although the markedly disturbed lipid homeostasis was observed in both HFD-OB and HFD-NOB rats (there were some distinctions in the elevations of factors involved in plasma lipid metabolism; Fig. 2), the different alterations in body weight increases and plasma lipid levels in the HFD-fed rats may partially explain why a certain incidence of cardiovascular diseases and hyperlipidemia exists in numerous individuals that are not overweight or obese as encountered in clinical practice. The changes in lifestyle (especially long-term high caloric intake), rather than only nutritional obesity, may be a major cause for AF incidence.

Along with tissue fibrosis, atrial enlargement is one of the two components of atrial structural remodeling (22). Atrial dimension has been demonstrated to be a determinant factor of the persistence of AF maintaining re-entry (23). In the present study, although no significant increase in body weight was observed in the HFD-NOB rats, the atrial weight was significantly higher (Fig. 1B). To some extent, this finding also suggested that chronic HFD feeding may lead to atrial enlargement which made the rats more susceptible to AF. In addition, distinctly broadened interstitial space among atrial myofibers induced by chronic HFD feeding (Fig. 3) may have contributed to promotion of the atrial enlargement. It has been confirmed in a previous study that tissue fibrosis could promote AF by interrupting atrial myocardium continuity and disturbing local conduction (24). Moreover, the interactions between fibroblasts and cardiomyocytes may give rise to changes in cardiomyocyte bioelectricity (25). Atrial fibrosis appears to be a common endpoint for a variety of AF-promoting elements (17). It has been acknowledged that TGF- $\beta 1$ is the key regulating factor of atrial fibrosis (18). It causes atrial fibrosis by activating downstream signaling pathways (26), and furthermore promotes the incidence and maintenance of AF. Moreover, MMP-2 appears to be another key factor involved in interstitial tissue alteration in diseased atrial myocardium (19,27). MMP-2 not only plays an important role in the degradation of the matrix, but also regulates the synthesis of collagen. Normal collagen is degraded by increased MMP-2, while it is replaced by fibrous interstitial that lacks connective structure. Moreover, the altered MMP/ TIMP equilibrium could result in a loss of control of MMP-2 activity and thereby contribute to an increase in MMP-2 activity in diseased atria. Finally, the fibrotic response strengthens as the MMP-2 levels increase. We analyzed Masson's staining, RT-qPCR and western blotting data to determine whether HFD induces atrial fibrosis in rats. In the present study, chronic HFD feeding increased fibrotic atrial myocardium (Fig. 4), upregulated expression of TGF- $\beta 1$ and MMP-2 protein and mRNA (Fig. 5), and these results were in accordance with the studies mentioned above. Upregulation in the expression of fibrosis-relevant factors revealed that the HFD induced marked fibrosis which may create the atrial substrate for re-entry. All these findings indicated that exposure to chronic HFD may promote AF by impacting both of the components of atrial structural remodeling, i.e., atrial enlargement and tissue fibrosis.

The essential role of gap junction in atrial conduction has been clearly described. Numerous studies in AF patients (28) and rapid pacing animal models $(29,30)$ have revealed the strong association between abnormal expression and heterogeneous distribution of Cxs and AF. Cx40 and Cx43, which mediate cardiomyocyte-to-cardiomyocyte electrical coupling, have been characterized as the major Cxs expressed in murine atria (31). Several animal studies $(15,16,32)$ displayed abnormality in expression and distribution of $\mathrm{Cx} 43$ in atria and ventricles following HFD feeding. Nevertheless, there are few studies elucidating the changes in expression and distribution of $\mathrm{Cx} 40$ in atria following HFD feeding. In our study, regardless of whether HFD feeding induced obesity, a significant 
decrease in expression of $\mathrm{Cx} 40$ and $\mathrm{Cx} 43$ protein and mRNA were observed in the HFD-fed rats (Fig. 6). Although the expression of $\mathrm{Cxs}$ in cardiac myocytes from animals fed an HFD have been studied $(15,16,32)$, discrepancies existed in these results. Notably, similarly in the present study, we found that $\mathrm{Cx} 40$ and $\mathrm{Cx} 43$ protein expression was distinctly upregulated in $1 / 5$ of the OB rats and in $1 / 8$ of the NOB rats which were opposite with the total expression levels (no significant difference was displayed between these two groups). However, no alteration in $\mathrm{Cx} 40$ and $\mathrm{Cx} 43$ expression was observed in any of the HFD rats. This finding may also support the fact that chronic HFD feeding impacts gap junction remodeling in atria. In addition, our analysis showed that $\mathrm{Cx} 40$ and $\mathrm{Cx} 43$ were more localized along longitudinal atrial myocyte membranes rather than the intercalated discs (Fig. 7). Spatial heterogeneities in distributions of Cxs may disturb the atrial conduction via creating microscopic obstacles altering the atrial action potential (33), which then gives rise to the incidence of AF. It therefore appears that HFD feeding may promote gap junction remodeling in atria, affect atrial myocyte action potential and atrial anisotropy conduction, thereby causing AF.

Apart from the AF-promoting conditions mentioned above, apoptosis cell death may be another potential mechanism contributing to AF by participating in atrial remodeling $(34,35)$. The effect of HFD-induced obesity on apoptosis in ventricles has been well documented $(20,21,36)$. In accordance with these results, we found a robust increase in cell death in both HFD-OB and HFD-NOB rat atria as detected by TUNEL assay (Fig. 8). The increase in the numbers of TUNEL-positive nuclei exhibited no difference in these two groups. We speculate that the alteration in cell apoptosis occurs in atrial myocytes of HFD-fed rats in the absence of obesity as well, thereby enhancing the atrial remodeling which may promote the incidence of AF.

However, there are several limitations to the present study. First, we measured atrial structure and gap junction remodeling, which are considered to be the main causes of AF. Although we determined the susceptibility to AF caused by chronic HFD, the incidence of AF was not examined. We may further investigate this aspect in the future. Second, the detailed mechanisms and the crosstalk between TGF- $\beta 1$, MMP-2 and gap junctions need further investigation.

We conclude that, regardless of the presence of HFD-induced obesity, an HFD promotes expression of factors involved in atrial fibrosis, altering the expression and distribution of Cxs in the atria, and inducing atrial myocyte apoptosis, which may further contribute to the incidence and development of AF. Thus, leading a healthy lifestyle by controlling calorie intake may be a promising therapeutic strategy for AF.

\section{Acknowledgements}

This study was supported by the Clinical Research Award of the First Affiliated Hospital of Xi'an Jiaotong University, Xi'an, China (no. XJTU1AF-CRF-2015-007).

\section{References}

1. Braunwald E: Shattuck lecture - cardiovascular medicine at the turn of the millennium: triumphs, concerns, and opportunities. N Engl J Med 337: 1360-1369, 1997.
2. Miyasaka Y, Barnes ME, Gersh BJ, Cha SS, Bailey KR, Abhayaratna WP, Seward JB and Tsang TSM: Secular trends in incidence of atrial fibrillation in Olmsted county, Minnesota, 1980 to 2000 , and implications on the projections for future prevalence. Circulation 114: 119-125, 2006.

3. Chamberlain AM, Agarwal SK, Ambrose M, Folsom AR Soliman EZ and Alonso A: Metabolic syndrome and incidence of atrial fibrillation among blacks and whites in the Atherosclerosis Risk in Communities (ARIC) study. Am Heart J 159: 850-856, 2010.

4. Ogden CL, Carroll MD, Kit BK and Flegal KM: Prevalence of childhood and adult obesity in the United States, 2011-2012. JAMA 311: 806-814, 2014.

5. Ng M, Fleming T, Robinson M, Thomson B, Graetz N, Margono C, Mullany EC, Biryukov S, Abbafati C, Abera SF, et al: Global, regional, and national prevalence of overweight and obesity in children and adults during 1980-2013: a systematic analysis for the Global Burden of Disease study 2013. Lancet 384: 766-781, 2014.

6. Mitchell S and Shaw D: The worldwide epidemic of female obesity. Best Pract Res Clin Obstet Gynaecol 29: 289-299, 2015.

7. Hill JO and Peters JC: Environmental contributions to the obesity epidemic. Science 280: 1371-1374, 1998.

8. Flegal KM, Graubard BI, Williamson DF and Gail MH: Excess deaths associated with underweight, overweight, and obesity. JAMA 293: 1861-1867, 2005.

9. Kahn SE, Hull RL and Utzschneider KM: Mechanisms linking obesity to insulin resistance and type 2 diabetes. Nature 444: 840-846, 2006.

10. Di Salvo G, Pacileo G, Del Giudice EM, Natale F, Limongelli G, Verrengia M, Rea A, Fratta F, Castaldi B, Gala S, et al: Atrial myocardial deformation properties in obese nonhypertensive children. J Am Soc Echocardiogr 21: 151-156, 2008.

11. Wong CY, O'Moore-Sullivan T, Leano R, Byrne N, Beller E and Marwick TH: Alterations of left ventricular myocardial characteristics associated with obesity. Circulation 110: 3081-3087, 2004.

12. Wang TJ, Parise H, Levy D, D'Agostino RB Sr, Wolf PA, Vasan RS and Benjamin EJ: Obesity and the risk of new-onset atrial fibrillation. JAMA 292: 2471-2477, 2004.

13. Abed HS, Samuel CS, Lau DH, Kelly DJ, Royce SG, Alasady M, Mahajan R, Kuklik P, Zhang Y, Brooks AG, et al: Obesity results in progressive atrial structural and electrical remodeling: implications for atrial fibrillation. Heart Rhythm 10: 90-100, 2013.

14. Mahajan R, Lau DH and Sanders P: Impact of obesity on cardiac metabolism, fibrosis, and function. Trends Cardiovasc Med 25: 119-126, 2015.

15. Aubin MC, Cardin S, Comtois P, Clément R, Gosselin H, Gillis MA, Le Quang K, Nattel S, Perrault LP and Calderone A: A high-fat diet increases risk of ventricular arrhythmia in female rats: enhanced arrhythmic risk in the absence of obesity or hyperlipidemia. J Appl Physiol 108: 933-940, 2010.

16. Axelsen LN, Calloe K, Braunstein TH, Riemann M, Hofgaard JP, Liang B, Jensen CF, Olsen KB, Bartels ED, Baandrup U, et al: Diet-induced pre-diabetes slows cardiac conductance and promotes arrhythmogenesis. Cardiovasc Diabetol 14: 87, 2015

17. Oakes RS, Badger TJ, Kholmovski EG, Akoum N, Burgon NS, Fish EN, Blauer JJE, Rao SN, DiBella EVR, Segerson NM, et al: Detection and quantification of left atrial structural remodeling with delayed-enhancement magnetic resonance imaging in patients with atrial fibrillation. Circulation 119: 1758-1767, 2009.

18. Verheule S, Sato T, Everett T IV, Engle SK, Otten D, Rubart-von der Lohe M, Nakajima HO, Nakajima H, Field LJ and Olgin JE: Increased vulnerability to atrial fibrillation in transgenic mice with selective atrial fibrosis caused by overexpression of TGF-beta1. Circ Res 94: 1458-1465, 2004.

19. Boixel C, Fontaine V, Rücker-Martin C, Milliez P, Louedec L, Michel JB, Jacob MP and Hatem SN: Fibrosis of the left atria during progression of heart failure is associated with increased matrix metalloproteinases in the rat. J Am Coll Cardiol 42: 336-344, 2003.

20. Dhahri W, Drolet MC, Roussel E, Couet J and Arsenault M: Chronic high-fat diet-induced obesity decreased survival and increased hypertrophy of rats with experimental eccentric hypertrophy from chronic aortic regurgitation. BMC Cardiovasc Disord 14: 123, 2014.

21. Wang HF, Lin PP, Chen CH, Yeh YL, Huang CC, Huang CY and Tsai CC: Effects of lactic acid bacteria on cardiac apoptosis are mediated by activation of the phosphatidylinositol-3 kinase/AKT survival-signalling pathway in rats fed a high-fat diet. Int J Mol Med 35: 460-470, 2015. 
22. Nattel S and Harada M: Atrial remodeling and atrial fibrillation: recent advances and translational perspectives. J Am Coll Cardiol 63: 2335-2345, 2014.

23. Zou R, Kneller J, Leon LJ and Nattel S: Substrate size as a determinant of fibrillatory activity maintenance in a mathematica model of canine atrium. Am J Physiol Heart Circ Physiol 289: H1002-H1012, 2005.

24. Burstein B, Comtois P, Michael G, Nishida K, Villeneuve L, Yeh YH and Nattel S: Changes in connexin expression and the atrial fibrillation substrate in congestive heart failure. Circ Res 105: 1213-1222, 2009.

25. Yue L, Xie J and Nattel S: Molecular determinants of cardiac fibroblast electrical function and therapeutic implications for atrial fibrillation. Cardiovasc Res 89: 744-753, 2011.

26. Rahmutula D, Marcus GM, Wilson EE, Ding CH, Xiao Y, Paquet AC, Barbeau R, Barczak AJ, Erle DJ and Olgin JE: Molecular basis of selective atrial fibrosis due to overexpression of transforming growth factor- $\beta 1$. Cardiovasc Res 99: 769-779, 2013

27. Rosenkranz S: TGF-beta1 and angiotensin networking in cardiac remodeling. Cardiovasc Res 63: 423-432, 2004.

28. Patel P, Jones D and Dupont E: Remodeling of human connexin 43 expression in human atrial fibrillation. Eur Heart J 19: 465, 2001.

29. Elvan A, Huang XD, Pressler ML and Zipes DP: Radiofrequency catheter ablation of the atria eliminates pacing-induced sustained atrial fibrillation and reduces connexin 43 in dogs. Circulation 96: 1675-1685, 1997.
30. van der Velden HM, Ausma J, Rook MB, Hellemons AJ, van Veen TA, Allessie MA and Jongsma HJ: Gap junctional remodeling in relation to stabilization of atrial fibrillation in the goat. Cardiovasc Res 46: 476-486, 2000.

31. Delorme B, Dahl E, Jarry-Guichard T, Briand JP, Willecke K, Gros D and Théveniau-Ruissy M: Expression pattern of connexin gene products at the early developmental stages of the mouse cardiovascular system. Circ Res 81: 423-437, 1997.

32. Mahajan R, Brooks A, Manavis J, Wood J, Finnie J, Sameul C, Lau D, Selvanayagam J, Roberts-Thomson K and Sanders P: Atrial fibrillation and obesity: impact of weight reduction on the atrial substrate. Heart Lung Circ 22: S1-S2, 2013.

33. Allessie M, Ausma J and Schotten U: Electrical, contractile and structural remodeling during atrial fibrillation. Cardiovasc Res 54: 230-246, 2002.

34. Gasparovic H, Cikes M, Kopjar T, Hlupic L, Velagic V, Milicic D, Bijnens B, Colak Z and Biočina B: Atrial apoptosis and fibrosis adversely affect atrial conduit, reservoir and contractile functions. Interact Cardiovasc Thorac Surg 19: 223-230, 2014.

35. Fu G, Cao Y, Lu J, Li J, Liu L, Wang H, Su F and Zheng Q: Programmed cell death-1 deficiency results in atrial remodeling in C57BL/6 mice. Int J Mol Med 31: 423-429, 2013.

36. Shi Z, Fu F, Yu L, Xing W, Su F, Liang X, Tie R, Ji L, $\mathrm{Zhu} \mathrm{M}, \mathrm{Yu}$ J, et al: Vasonatrin peptide attenuates myocardial ischemia-reperfusion injury in diabetic rats and underlying mechanisms. Am J Physiol Heart Circ Physiol 308: H281-H290, 2015. 